\title{
El Centro de Capacitación en Educación a Distancia: de la capacitación al desarrollo profesional $2000-2010$
}

\section{The Centro de Capacitación en Educación a Distancia: from training to professional development $2000-2010$}

\author{
Warner Ruiz Chaves \\ Capacitador del Centro de Capacitación en Educación a Distancia \\ Universidad Estatal a Distancia \\ San José, Costa Rica \\ wruiz@uned.ac.cr
}

Recibido: 20-IX-2010 • Aceptado 19-X-2010 • Corregido 14-IV-2011

\begin{abstract}
Resumen: El escrito presenta una breve sistematización de la experiencia del Centro de Capacitación en Educación a Distancia (CECED) de la Universidad Estatal a Distancia (UNED) de Costa Rica con el fin de comprender la evolución de la concepción de capacitación "tradicional" a una propuesta de desarrollo profesional docente en el cual los equipos académicos de la Universidad se comprometieron no solo con la mejora personal sino en vivir la experiencia de la Misión y la Visión y el Modelo Pedagógico centrado en el estudiante que tiene la Institución. Esto de modo que se evidencien prácticas tutoriales de calidad, es decir, buscar la excelencia académica para la construcción de una sociedad justa basada, entre otros aspectos, en una exigencia académica en sus quehaceres fundamentales: docencia, investigación, extensión $y$ producción de materiales didácticos. Todo con el objetivo de alcanzar los niveles educativos superiores deseados en condiciones de calidad, pertinencia $y$ equidad, acordes con las demandas de los diversos grupos de la sociedad costarricense. Para ello se analizaron las estrategias metodológicas aplicadas en algunos cursos y los comentarios de participantes de las experiencias.
\end{abstract}

\section{Presentación}

El contexto mundial ha modificado a la humanidad y del mismo modo ha transformado la educación, la cual ocupa un lugar relevante en la historia humana, particularmente la educación superior del siglo XXI ante los estudiantes, pues debe promover aquellos saberes que proponía Jacques Delors (1996): el aprender a hacer, aprender a ser, aprender a conocer y aprender a convivir.

En este sentido, la Universidad Estatal a Distancia (UNED) de Costa Rica ha dirigido esfuerzos para presentar en las políticas institucionales y en su Modelo Pedagógico este paradigma (centrado en quien aprende), en el cual los estudiantes no solo tratan de desarrollar esos saberes, sino que los profesionales que desempeñan 
Palabras clave: Desarrollo profesional docente, educación, capacitación, educación a distancia.

\begin{abstract}
This paper presents a brief systematization of the experiences of the Centro de Capacitacion en Educación a Distancia (CECED) from the Universidad Estatal a Distancia (UNED) of Costa Rica with the goal to understand the evolution of the "traditional" training concept, to a professional development concept. This proposal is directed toward students and educators who want to improve their capabilities through the application of our mission and vision and the Pedagogical Model. CECED has been looking for excellence in academic areas by using tutorial practices where the student is the most important part of the equation. Students can benefit from the experience of learning furthermore from our society and acquire better educational standards, therefore providing more opportunities for everyone. Thus, CECED provides both self-confidence and personal development. The program's goal can be increased by using elements such as equality, pertinence and equity to make the teaching learning process more effective. It will improve motivation to develop competition in the general curriculum and simultaneously result in greater proficiency in the target group. We have analyzed this through situations and comments from our students to develop the strategies.
\end{abstract}

Keywords: Professional teacher development, Education, Training, Distance learning cargos docentes se desenvuelven y se actualizan en torno a esta dirección. No obstante, no siempre esta noción se tuvo tan clara como se tiene en este momento.

La importancia de la capacitación docente fue asumida por la UNED en la sesión del Consejo de Rectoría 11422000 del día 15 de mayo de 2000, cuando se avaló la creación del Centro de Capacitación en Educación a Distancia (CECED) como dependencia adscrita a la Vicerrectoría Académica.

A partir de este momento, se gestaron diferentes procesos de capacitación que, con el paso de los años y la actualización en tendencias educativas, ha devenido en la implementación de procesos de desarrollo profesional, los cuales atienden de forma sistemática las necesidades de los equipos docentes de la UNED. Por esta razón, documentar la experiencia acumulada por el CECED en sus diez años de creación representa una manera de aportar a la comunidad académica las buenas prácticas de este Centro y contribuir con la mejorar del quehacer universitario.

El presente escrito versa sobre tres áreas desarrolladas de forma conexa: una muy breve historia relativa a la capacitación docente en la UNED, particularmente referida al CECED; un análisis de diferentes procesos de formación visualizando su propósito, actividades, estrategias y evaluaciones para comprender cómo el CECED ha evolucionado hasta promover realmente un desarrollo profesional; y por último, las experiencias de los participantes de diferentes procesos formativos que ratifican la importancia del desarrollo profesional como un factor de éxito para su práctica tutorial y personal. Se pretende entonces como objeto de estudio de este escrito comprender la evolución de la concepción de capacitación tradicional que desarrollaba el CECED a una propuesta de desarrollo profesional docente, con el fin de visualizar cómo ha incidido en los equipos docentes de la UNED. 
Se desarrolla una metodología basada en el análisis comparativo de tres períodos desde la creación del CECED: 20002004, 2005-2006, 2007-2010. A partir de ello se toman los cursos que mayormente se implementaron y se transcribe íntegramente su propósito u objetivo, así como las metodologías y estrategias de los mismos con el fin de poder visualizar los avances o retrocesos en las concepciones de capacitación a los largo de los 10 años de creación.

Se hace una breve alusión a referentes conceptuales sobre los que se parte, sin necesidad de asumir una concepción a priori de los mismos, pues la riqueza de la sistematización de este escrito es que permite servir de insumo para una reflexión sobre el quehacer del Centro y sus prácticas desde fuentes primarias: programas de cursos y percepciones de los participantes. Sin embargo, no se debe perder de vista que el Centro de Capacitación hace suyos los postulados del Modelo Pedagógico de la UNED, de modo que desde sus inicios, en algunos casos más en otros menos, se ubican en la práctica las actuales bases teóricas del Modelo.

\section{Desafío institucional y necesidades atendidas}

El desarrollo profesional docente es un elemento clave para la mejora del sistema educativo (Culp, Kanaya \& Light, 2005; Dede, 2006; Guskey, 2000). En este sentido, la necesidad de mejorar los procesos de actualización de tutores en la UNED, tanto de aspectos pedagógicos como de contenidos disciplinares y habilidades tecnológicas ha sido identificada como una de las acciones vitales para facilitar un cambio dentro de la Universidad (Castillo, 2010).

Por lo anterior, surge como inquietud y necesidad el consolidar el ámbito de conocimiento tanto práctico como teórico de los docentes. De acuerdo con Noguera, Fuentealba, Osandón, Portales y Quiroga (2002):
Las múltiples aproximaciones hoy existentes en torno a cómo mejorar dicha situación, en su mayoría asumen que el trabajo docente se relaciona con el manejo de un conjunto de técnicas. Es decir, el desarrollo profesional es concebido como un proceso acumulativo de técnicas y procedimientos, en el cual el profesor en forma aislada, se reduce a un consumidor o destinatario de todos estos conocimientos producidos por unos expertos, ajenos a la realidad donde el docente se desempeña (p.13).

Al revisar la literatura, se coincide con Fallas, Arias, Villers, Escalante y McCloskey (2010) en que el tema del desarrollo profesional para docentes presenta aspectos problemáticos que han caracterizado su ejecución:

"Los cursos de capacitación que se ofrecen tienden a ser de carácter puntual y breve; no se basan en resultados de investigación y tampoco remiten a procesos rigurosos de formación permanente (Dede, Ketelhut, Whitehouse, Breit \& McCloskey, 2009). Al respecto, Borko (2004) indica que el desarrollo profesional es lamentablemente inadecuado, fragmentado, intelectualmente superficial y no toma en cuenta lo que se conoce sobre la forma en la que los educadores aprenden.

- La mayoría de las actividades que se ofrecen bajo el nombre de desarrollo profesional tienden a ser experiencias aisladas y desarticuladas y no contemplan un seguimiento durante la implementación de las propuestas por parte del docente en el aula, lo que ha comprobado ser un requisito para influir positivamente la práctica docente (Barnett, Keating, Harwood y Saam, 2002). La carencia de procesos que conducen a la reflexión, sobre todo entre elementos de la teoría y la práctica en el aula, limitan la sostenibilidad de los cursos actuales 
en el tiempo (Marzano, 2003).

- Es posible identificar una fuerte tendencia a saturar los cursos con teorías supuestamente de moda mundial, con múltiples neologismos o tecnicismos que oscurecen el lenguaje y la comprensión del mensaje. En general, los maestros regresan a sus aulas con nuevas categorías pero con las mismas prácticas. Sin embargo, no siempre sucede así (CIDE, 2001).

- Las agendas sobrecargadas de los tutores y la dificultad de asignarles espacios para su capacitación constituyen serias debilidades para este sector (Robalino, 2004) (pág. 2-3)".

Por ello, a pesar de hablar de desarrollo profesional docente, en realidad la mayoría de las veces, tanto la UNED, como otros entes de formación continuaban ofreciendo la capacitación "tradicional", entendida esta como lo propone Huberman (2005): "un proceso consciente (...) y deliberado implementado por un sistema educativo o una organización con el objeto de mejorar desempeños y resultados (...)". Por ello, la concepción actual de desarrollo profesional volcada hacia esa mejora continua del profesional en donde él piense, juzgue, valore y actúe no se visualizaba ni remotamente en los primeros años del CECED.

Por su lado, el Modelo Pedagógico de la Universidad propone al participante de los procesos formativos como el elemento central del proceso de enseñanzaaprendizaje, pues es a partir de él que el modelo desarrolla su práctica generando una interacción con los componentes del mismo Modelo, a saber: los contenidos, el medio, los tutores y la relación con otros participantes. Sin embargo, esta propuesta pedagógica nace en la UNED en el 2004, de modo que es hasta este momento en que los procesos de capacitación ofrecidos por el CECED tratan de incluirla y experimentarla.
Esta innovadora propuesta pedagógica se fundamenta en la Teoría de Construcción Social del Aprendizaje, la cual está ligada a la Teoría del Aprendizaje Cognitivista, que señala que el individuo está sometido e inmerso en determinadas situaciones que hacen que la cognición sea una interpretación significativa de la interacción social con el mundo que lo rodea; interpretación de un determinado reforzamiento y de las conexiones realizadas respecto de una experiencia vivida. Según Salas (2009), lo anterior es un ejemplo que demuestra que los procesos de aprendizaje pueden ser significativos. Ausubel (citado por Ander Egg, 1993), afirma que "el factor más sencillo y más importante que influye en el aprendizaje es lo que ya sabe el que aprende". En este caso, la capacitación "tradicional" fue cambiando poco a poco en las experiencias formativas del CECED, conforme se fue aplicando el Modelo Pedagógico.

\section{La capacitación docente en la UNED: contextualización del aporte del CECED a los procesos de enseñanza- aprendizaje en educación a distancia}

El CECED tiene sus antecedentes en el Programa de Didáctica Universitaria. Este programa se creó en 1989 como parte de la Vicerrectoría Académica de la UNED, bajo la iniciativa del M.Ed. Fernando Castro y fue dirigido por el Dr. Héctor Mejía. En 1991, se trasladó a la Oficina de Programación Curricular bajo la coordinación de la Dra. Delfilia Mora, para ser incorporado tiempo después a la Escuela de Educación.

En el mismo año, se reformuló el Programa en atención a la Política Institucional aprobado por la Asamblea Universitaria de la Universidad Estatal a Distancia de Costa Rica en 1990, lo cual 
promovió la producción de estímulos y condiciones apropiados para que el personal académico pudiera cumplir con los fines de investigación, docencia y extensión.

Es de este modo que el Programa buscó coadyuvar con el logro de la excelencia académica, a partir de la reflexión del docente a distancia acerca de su propio quehacer y utilizando algunos elementos teórico-prácticos, tendientes a mejorar las habilidades de planificación, programación, ejecución y evaluación de los procesos de aprendizaje.

Con el paso de los años, se observó la necesidad de que este Programa fuera fortalecido y se reestructurara en función de lo solicitado por el II Congreso Universitario (máximo órgano de la Institución) celebrado en el año 2000. Es así como se creó el Centro de Capacitación en Educación a Distancia, por acuerdo del Consejo de Rectoría del 15 de mayo de ese mismo año, con el fin de incentivar en el docente universitario un análisis y reflexión sobre sus propias prácticas para que mejore no solo su desempeño, sino el impacto de la Universidad en la sociedad.

El proyecto del CECED surgió como iniciativa del rector de ese entonces, Rodrigo Arias Camacho (2000-2009). Su primera coordinadora fue la Dra. Delfilia Mora Hamblin por espacio de cuatro años (2000-2004), a quien le sucede la Magíster Johanna Meza Vargas (2004-2006) y actualmente, es dirigido por la Magíster Marianela Salas Soto.

El Centro tiene como Misión, según Mora (2000):

Ser una dependencia especializada en capacitación académica, que trata de incentivar al profesional universitario para que reflexione críticamente y aplique sobre sus experiencias docentes de cara a los avances en el campo de la pedagogía, la didáctica y de las nuevas metodologías de la enseñanza a distancia, mejorando habilidades, principalmente en lo referente a la planificación, programación, ejecución y evaluación del proceso de enseñanza y aprendizaje (pág. 3).
En su visión, de acuerdo con la propuesta de Mora (2000):

El CECED busca ser un centro de capacitación que proyecte una regionalización de la capacitación en educación a distancia, una participación eficaz de sus profesionales en las políticas educativas, una construcción permanente de la pedagogía universitaria en un proceso de transformación en busca la mejora de la calidad educativa, en beneficio de los responsables en la entrega de la docencia en la UNED como un todo, y de la realidad social, cultural y económica del país (pág. 4).

Tomando como referencia lo anterior, durante los primeros años del CECED, la actividad se centró en el desarrollo de un curso que ayudara a comprender la actividad docente de la UNED: Didáctica universitaria, el cual, según su coordinadora, Delfilia Mora (Mora, D. entrevista personal realizada el 28 de abril de 2010), se llevó a cabo de manera presencial con otros especialistas externos debido a las carencias de personal del Centro. De hecho, hasta la asignación de profesionales docentes a esta dependencia es que se empezó a diversificar la oferta, pensando no solo en los centros universitarios, sino en cursos para entidades extranjeras.

Así, entonces, en esta etapa de funcionamiento del Centro las acciones giraron en torno a una concepción de capacitación referida al proceso consciente y dirigido implementado por la dependencia hacia los tutores, encargados de cátedra y productores académicos de la UNED para mejorar su desempeño.

Las primeras experiencias, todas de modalidad presencial, fueron procesos en los que un grupo de académicos especialistas exponían de manera magistral una temática y los participantes realizaban trabajo cooperativo.

Obsérvese el siguiente cuadro con algunas estrategias aplicadas en los cursos. Este cuadro refleja cómo la concepción de una capacitación dirigida de forma "tradicional" fue predominante durante el período 2000-2004, ya que, como pudo 
Tabla 1

Estrategias aplicadas en algunos cursos del CECED durante el período 2000-2004

\begin{tabular}{|c|c|c|c|}
\hline Curso / Año & Objetivo o propósito & $\begin{array}{l}\text { Estrategia } \\
\text { metodológica }\end{array}$ & Técnicas didácticas \\
\hline $\begin{array}{l}\text { Didáctica } \\
\text { Universitaria } \\
(2000-2001)\end{array}$ & $\begin{array}{l}\text { Analizar la importancia } \\
\text { de la pedagogía como } \\
\text { disciplina científica y su } \\
\text { aporte en los procesos de } \\
\text { la enseñanza y el apren- } \\
\text { dizaje en la educación a } \\
\text { distancia. }\end{array}$ & $\begin{array}{l}\text { No se encontraron los } \\
\text { datos. }\end{array}$ & $\begin{array}{l}\text { Trabajo individual } \\
\text { Proceso de análisis y síntesis } \\
\text { Devoluciones sobre lo apren- } \\
\text { dido } \\
\text { Confrontación entre lo que yo } \\
\text { he hecho y lo que espero hacer } \\
\text { Aportes de mi propia práctica } \\
\text { docente. }\end{array}$ \\
\hline $\begin{array}{l}\text { Elaboración de } \\
\text { programas de } \\
\text { curso } \\
(2002)\end{array}$ & $\begin{array}{l}\text { Conocer las principales } \\
\text { características de los } \\
\text { modelos de diseño de } \\
\text { programas de curso que } \\
\text { podrían utilizarse en la } \\
\text { educación a distancia. }\end{array}$ & $\begin{array}{l}\text { Se pretende mantener } \\
\text { una relación dialógica } \\
\text { continua con los parti- } \\
\text { cipantes que permita la } \\
\text { construcción individual } \\
\text { y social del conocimien- } \\
\text { to. Por la naturaleza del } \\
\text { taller, los participantes } \\
\text { elaborarán una propuesta } \\
\text { de programa de curso que } \\
\text { responda a las particu- } \\
\text { laridades institucionales. }\end{array}$ & $\begin{array}{l}\text { Exposición magistral del } \\
\text { especialista } \\
\text { Intercambio de opiniones } \\
\text { sobre la temática y su perti- } \\
\text { nencia institucional } \\
\text { Elaboración de programas de } \\
\text { curso por parte de los parti- } \\
\text { cipantes }\end{array}$ \\
\hline $\begin{array}{l}\text { La investigación } \\
\text { cualitativa } \\
(2003)\end{array}$ & $\begin{array}{l}\text { Generar y construir cono- } \\
\text { cimiento desde la investi- } \\
\text { gación cualitativa en los } \\
\text { procesos académicos de } \\
\text { la educación a distancia. }\end{array}$ & $\begin{array}{l}\text { Se desarrolla mediante } \\
\text { la modalidad taller y se } \\
\text { adopta el diálogo como } \\
\text { método para construir } \\
\text { los argumentos y con- } \\
\text { clusiones a que se llegue } \\
\text { durante las sesiones pre- } \\
\text { senciales. }\end{array}$ & $\begin{array}{l}\text { Exposición magistral del } \\
\text { especialista } \\
\text { Intercambio de opiniones } \\
\text { sobre la temática y su prácti- } \\
\text { ca docente }\end{array}$ \\
\hline $\begin{array}{l}\text { Estrategias } \\
\text { docentes para la } \\
\text { educación a } \\
\text { distancia } \\
(2004)\end{array}$ & $\begin{array}{l}\text { Bridar los fundamentos } \\
\text { de la educación a dis- } \\
\text { tancia, sus estrategias } \\
\text { didácticas y de evalua- } \\
\text { ción, desde una perspec- } \\
\text { tiva integrada y haciendo } \\
\text { énfasis en el rol del tutor } \\
\text { dentro de la intervención } \\
\text { y mediación pedagógica } \\
\text { en la tutoría. }\end{array}$ & $\begin{array}{l}\text { Se fundamenta en el } \\
\text { aporte teórico que el espe- } \\
\text { cialista ofrece sobre el } \\
\text { tema, el cual es insumo } \\
\text { para generar la discusión } \\
\text { sobre la temática y luego, } \\
\text { los participantes confron- } \\
\text { tan sus experiencias para } \\
\text { realizar una síntesis. }\end{array}$ & $\begin{array}{l}\text { Exposición magistral del } \\
\text { especialista } \\
\text { Intercambio de opiniones } \\
\text { sobre la temática y su prácti- } \\
\text { ca docente }\end{array}$ \\
\hline
\end{tabular}

Fuente: Elaboración propia con base en los diseños didácticos de los cursos de la dependencia. Se reproducen fielmente lo señalado en los diseños.

verse, sobresalían en todos los casos las exposiciones magistrales de especialistas en áreas como la evaluación de los aprendizajes, la educación de adultos, la educación a distancia, el medio ambiente, la investigación, la educación y el género, los medios didácticos y el diseño curricular, entre otros.

Desde este enfoque, se podría afirmar que se hablaba de una capacitación 
"tradicional" que trataba de ofrecer a los equipos docentes una serie de informaciones o conocimientos necesarios para que su desempeño en la Universidad fuera contextualizado de manera eficaz y eficiente. Algunas características que se desprenden del análisis de los programas de curso en este período son:

- En la mayoría de las ocasiones, las convocatorias fueron de carácter obligatorio, pues se condicionaba la continuidad en el cargo, siempre y cuando las personas se capacitaran.

- No se brindaba una centralidad al participante, sino que este era sólo un receptor de información que podía, eventualmente, reproducirla de manera eficiente.

- No se visualizaban los aspectos motivacionales intrínsecos del proceso de aprendizaje. En este sentido, el deseo de superación personal no siempre estaba presente.

- No se utilizaba el trabajo colaborativo como técnica para promover una capacitación más provechosa y funcional para quien aprende.

- Los cursos fueron solamente presenciales.

- Los logros durante la primera etapa del CECED (2000-2004) se pueden identificar como:

- La definición organizativa y las fun- ciones del Centro.

- La concreción de presupuesto, equipo e infraestructura propios, dejando de utilizar los de la Escuela de Educación.

- Las visitas a los Centros Universitarios para brindar capacitación en el mismo lugar, evitando que los tutores se tuvieran que trasladar.

- La obligatoriedad del curso de Didáctica universitaria para todo docente de la UNED.
En suma, se puede determinar que durante este período, las estrategias utilizadas correspondieron a una concepción clásica de capacitación, en la cual los especialistas de las temáticas eran quienes "tenían la batuta" del proceso; es decir, el proceso se centraba más en quien enseñaba que en quien aprendía (contrario a lo que vendría a proponer el Modelo Pedagógicos años después). En este sentido, la presencialidad de los cursos fomentaba esto, pues predominaban las presentaciones magistrales.

Ahora bien, para comprender la interiorización del período, estrategias y curso, se presentan las experiencias de tres personas con respecto a los procesos que recibieron en el período:

Tabla 2

Comentarios de los participantes en cursos del CECED durante el período 2000-2004

\begin{tabular}{ll}
\hline Aspectos positivos & Aspectos por mejorar \\
\hline - Conocer a & - Mayor interacción y \\
compañeros de otras & aprendizaje con otros \\
dependencias. & compañeros. \\
- Saber qué hacen en & - Más actividades. \\
las otras & - Aumentar la variedad \\
dependencias. & de la oferta. \\
- Intercambio de & - El tiempo destinado \\
experiencias entre & para las sesiones pre- \\
distintas cátedras. & senciales es muy poco, \\
- Discusión y & a veces lo que explican \\
propuestas sobre los & lo hacen tan rápido que \\
problemas y realida- & uno se pierde. \\
des de la UNED. & - No conocer las carac- \\
- Conocimiento de & terísticas del grupo \\
recursos para la & meta y por ende, no \\
educación a distancia. & haber contextualizado \\
- Libros muy claros y & de manera pertinente el \\
de diferentes & proceso de capacitación. \\
temáticas. & - El poco apoyo de las \\
& autoridades con res- \\
& pecto a los procesos de \\
& capacitación. \\
\hline
\end{tabular}

Fuente: Ruiz, Warner (2010). Encuesta sobre la percepción de los participantes de los cursos ofrecidos por el CECED durante los años 2000-2010. Manuscrito no publicado. 
Durante la segunda etapa (20042006), el interés del CECED se concentró en diversificar la oferta formativa, introduciéndose en la capacitación mediante los entornos virtuales. Del mismo modo, según su coordinadora Johanna Meza (Meza, J. entrevista personal, 20 de abril de 2010), se repensó tanto la metodología con que se desarrollaban los cursos, así como la producción de materiales propios y la evaluación de los aprendizajes, todo orientado hacia la mejora continua.

La idea fundamental de los procesos de capacitación llevados a cabo giró en torno a que los logros de una institución, y sobre todo de una institución educativa, se gestan en entornos de personas con un conocimiento científico y tecnológico permanentemente actualizado.

De este modo, se inició una oferta más variada y ajustada a los cambios que señalaba la sociedad de la información y la comunicación. En este sentido, se implementó la utilización de entornos virtuales para el aprendizaje de los participantes de los cursos. No obstante, no se trataba solo de una innovación desde lo tecnológico, sino de una complementariedad con lo pedagógico.

Según Meza (2010), este período inició con Microcampus; sin embargo, por los inconvenientes que presentaba, se gestionó la utilización del Portal Educativo de las Américas de la Organización de los Estados Americanos. En el 2005, la Universidad adquirió el Learning Management System (LMS) propietario: Web-CT.

De forma puntual, algunas características de este período y que se pueden detallar en el cuadro número tres son:

Realización de trabajos colaborativos en línea, lo cual potenciaba el desarrollo de la competencia y el medio.

Ampliación de la oferta formativa con cursos no solamente teóricos, sino prácticos, relacionados con el desarrollo de cursos en línea, el tutor virtual, la producción de materiales didácticos, entre otros.
Reducción de las visitas a los centros universitarios y el proyecto de videoconferencia.

Incorporación de los elementos del Modelo Pedagógico de la UNED como elemento central en todo proceso formativo, es decir, promover la centralidad del estudiante y su interrelación con los contenidos, medios, tutores y otros estudiantes de una manera más "horizontal" y participativa.

Se propició la creación de un sentido de pertenencia a la Universidad mediante las dinámicas propuestas.

Todo esto implicó que el Centro rediseñara sus estrategias didácticas, en función de promover aprendizajes más colaborativos e innovadores, propios de los medios virtuales. En el anterior sentido, Salas (2009) señala que la oferta de cursos del Centro se conceptualizó:

Como un conjunto de actividades (talleres, cursos, seminarios, conversatorios), las cuales permitirían al personal docente de la institución, reflexionar sobre el uso que se le ha dado a la virtualidad $y$, a la vez, recrear una práctica educativa en línea que realmente ofreciera garantía de éxito en los procesos de enseñanza- aprendizaje. Para ello en todo momento, se estimuló la reflexión, el debate y el análisis de experiencias, las cuales enriquecieran el diseño de un programa o de un proyecto concreto de mejoramiento de la calidad educativa en entornos virtuales (p.3).

Como se nota en el cuadro número dos, las técnicas promovieron el análisis de experiencias, la reflexión de la práctica docente y el debate entre iguales para la mejora personal e institucional. Por ello, los principales logros se pueden resumir así:

La reformulación del curso Didáctica universitaria por Pedagogía universitaria en la educación a distancia, aprovechando insumos como el Modelo Pedagógico, la centralidad del estudiante, la evaluación de los aprendizajes y el diseño curricular.

La utilización de plataformas de aprendizaje en línea, como por ejemplo la de la OEA, Microcampus y posteriormente, WebCT. 
La ampliación de la oferta formativa, ya no sólo con cursos, sino con conferencias, talleres y mesas redondas.

El uso del correo electrónico institucional para la formulación de retos pedagógicos a los funcionarios sobre temas relacionados con la educación a distancia.
A grandes rasgos, el segundo período es importante pues se dio una redefinición de las metas del CECED, dado que los procesos de capacitación se concibieron como indispensables para la consecución de los objetivos de la Institución. Lo anterior, debido a que los profesores tutores que

Tabla 3

Estrategias aplicadas en algunos cursos del CECED durante el período 2005-2006

\begin{tabular}{|c|c|c|c|}
\hline Curso / Año & Objetivo o propósito & $\begin{array}{c}\text { Estrategia } \\
\text { metodológica }\end{array}$ & Técnicas didácticas \\
\hline $\begin{array}{l}\text { Pedagogía } \\
\text { universitaria } \\
\text { para la educación } \\
\text { a distancia } \\
(2005)\end{array}$ & $\begin{array}{l}\text { Comprender el concepto } \\
\text { de educación a distan- } \\
\text { cia, a fin de valorar su } \\
\text { interdependencia con el } \\
\text { logro de la calidad en el } \\
\text { proceso de enseñanza- } \\
\text { aprendizaje. }\end{array}$ & $\begin{array}{l}\text { Se propicia una reflexión } \\
\text { de la práctica docente } \\
\text { buscando el mejoramien- } \\
\text { to de la docencia en nues- } \\
\text { tro sistema de educación } \\
\text { a distancia, de modo que } \\
\text { se estudien métodos, téc- } \\
\text { nicas y medios que se } \\
\text { adapten a la realidad y } \\
\text { a las características de } \\
\text { nuestros estudiantes e } \\
\text { institución. }\end{array}$ & $\begin{array}{l}\text { - Sesiones presenciales donde el } \\
\text { especialista interactúa con los } \\
\text { participantes } \\
\text { - Interacción en los foros en } \\
\text { línea } \\
\text { - Desarrollo de un portafolio }\end{array}$ \\
\hline $\begin{array}{l}\text { Evaluación de los } \\
\text { aprendizajes } \\
(2006)\end{array}$ & $\begin{array}{l}\text { Propiciar la construcción } \\
\text { y el uso de estrategias } \\
\text { evaluativas que contribu- } \\
\text { yan al mejoramiento del } \\
\text { proceso de enseñanza- } \\
\text { aprendizaje, de acuerdo } \\
\text { con el Modelo Pedagógico } \\
\text { de la UNED. }\end{array}$ & $\begin{array}{l}\text { Propicia la construc- } \\
\text { ción de los aprendizajes } \\
\text { y conocimientos, forta- } \\
\text { leciendo las destrezas y } \\
\text { actitudes necesarias para } \\
\text { el desarrollo de un pro- } \\
\text { ceso de evaluación acor- } \\
\text { de con el propuesto por } \\
\text { la Universidad, para lo } \\
\text { cual se abren espacios de } \\
\text { discusión para la parti- } \\
\text { cipación reflexiva en las } \\
\text { sesiones presenciales y } \\
\text { virtuales. }\end{array}$ & $\begin{array}{l}\text { - Exposición magistral del } \\
\text { especialista } \\
\text { - Intercambio de opiniones } \\
\text { sobre la temática y su perti- } \\
\text { nencia institucional } \\
\text {-Interacción en los foros en } \\
\text { línea } \\
\text { - Desarrollo de un portafolio } \\
\text { - Reelaboración de textos } \\
\text {-Construcción de estrategias } \\
\text { alternativas }\end{array}$ \\
\hline
\end{tabular}

Fuente: Elaboración propia con base en los diseños didácticos de los cursos de la dependencia. Se reproducen fielmente lo señalado en los diseños. 
recibieron cursos como los que ofrecía el Centro aprendieron no solo nuevas teorías y nuevas posibilidades o tecnologías que les ayudaban a ser mejores docentes y personas, sino también a compartir experiencias, reflexionar y crear nuevos conocimientos de forma colaborativa. Esto hizo que sus estudiantes también se beneficiaran al compartir los aprendizajes del profesor. Con respecto a la los participantes en los cursos, estos señalaron:

Tabla 4

Comentarios de los participantes en cursos del CECED durante el período 2005-2006

\begin{tabular}{|c|c|}
\hline Aspectos positivos & Aspectos por mejorar \\
\hline $\begin{array}{l}\text { - En el curso de estrategias a distancia, la meto- } \\
\text { dología utilizada fue muy valiosa, el trabajo y la } \\
\text { exigencia de producción muy profesional por parte } \\
\text { de los y las capacitadoras. } \\
\text { - Socializar con otros compañeros de la UNED, mi } \\
\text { primera experiencia en cursos en línea. } \\
\text { - Trabajo en equipo con los cursos a través de la } \\
\text { plataforma. }\end{array}$ & $\begin{array}{l}\text { - Poco acompañamiento en el proceso, por ejemplo } \\
\text { el curso de investigación. } \\
\text { - En algunas ocasiones los programas no abren y } \\
\text { eso provoca estrés, algunas veces poco tiempo para } \\
\text { realizar los diferentes aportes. } \\
\text { - Poca paciencia de los tutores, dudas no contesta- } \\
\text { das, indicaciones no claras. }\end{array}$ \\
\hline $\begin{array}{l}\text { - En cuestión de tiempo, me ha permitido obte- } \\
\text { ner mayor provecho, agilizando la revisión de } \\
\text { trabajos o documentos o aclarando dudas de los } \\
\text { estudiantes. }\end{array}$ & \\
\hline $\begin{array}{l}\text { - Ha sido muy satisfactorio porque aspectos que } \\
\text { desconocía, con la capacitación llegué a aplicar } \\
\text { mejor las herramientas y conocimientos adqui- } \\
\text { ridos. }\end{array}$ & \\
\hline $\begin{array}{l}\text { - Unión de grupo, descubrimiento de capacidades, } \\
\text { compartir con distintos profesionales. }\end{array}$ & \\
\hline
\end{tabular}

Fuente: Ruiz, Warner (2010). Encuesta sobre la percepción de los participantes de los cursos del CECED durante los años 2000-2010. Manuscrito no publicado.

Por último, en la tercera y actual etapa (2007-2010), hay una redefinición de las propuestas formativas que se habían venido implementando, así como una diversificación.

En este sentido, según la coordinadora, Marianela Salas (Salas, M. entrevista personal, 25 de abril de 2010), aparte de lo anterior, se logró tener más profesionales dedicados a la promoción del desarrollo profesional y mejores equipos de cómputo para el personal y para la implementación de los cursos.

Igualmente, se pudo diversificar la oferta de cursos de manera que no solo se atendiera lo pedagógico, sino también la parte tecnológica, lo administrativo, lo curricular y la investigación, todos quehaceres propios de la Academia. Nótese la siguiente tabla para observar las estrategias: 
Tabla 5

Estrategias aplicadas en algunos cursos del CECED durante el período 2007-2010

\begin{tabular}{|c|c|c|c|}
\hline Curso / Año & Objetivo o propósito & $\begin{array}{l}\text { Estrategia } \\
\text { metodológica }\end{array}$ & Técnicas didácticas \\
\hline $\begin{array}{l}\text { La tutoría } \\
\text { virtual } \\
(2007)\end{array}$ & $\begin{array}{l}\text { Analizar los elementos } \\
\text { teóricos que implican } \\
\text { la gestión y moderación } \\
\text { de un curso virtual, así } \\
\text { como las funciones del } \\
\text { tutor virtual. }\end{array}$ & $\begin{array}{l}\text { Se busca que las y los parti- } \\
\text { cipantes conozcan los elemen- } \\
\text { tos necesarios para le gestión } \\
\text { y moderación de un curso vir- } \\
\text { tual, por eso la modalidad } \\
\text { será completamente virtual. }\end{array}$ & $\begin{array}{l}\text { - Interacción en los foros en } \\
\text { línea } \\
\text { - Simulación de un foro }\end{array}$ \\
\hline $\begin{array}{l}\text { Enseñar y } \\
\text { aprender en } \\
\text { entornos } \\
\text { virtuales } \\
\text { (2008) }\end{array}$ & $\begin{array}{l}\text { Facilitar que los docen- } \\
\text { tes se involucren en la } \\
\text { utilización de los entor- } \\
\text { nos virtuales y todas } \\
\text { las dimensiones peda- } \\
\text { gógico-didácticas que } \\
\text { implican, dentro de la } \\
\text { modalidad de educación } \\
\text { a distancia. De esta } \\
\text { manera, deberán con- } \\
\text { vertirse en elementos } \\
\text { que permitan el mejo- } \\
\text { ramiento de la calidad } \\
\text { del sistema educativo } \\
\text { de la UNED en cual- } \\
\text { quiera de sus niveles, } \\
\text { mediante la formula- } \\
\text { ción de reformas, pro- } \\
\text { puestas de programas, } \\
\text { planes, proyectos de } \\
\text { cursos o carreras en } \\
\text { línea. }\end{array}$ & $\begin{array}{l}\text { La naturaleza teórico-prácti- } \\
\text { ca del curso plantea involu- } \\
\text { crar a los estudiantes en un } \\
\text { proceso cooperativo de apren- } \\
\text { dizaje a partir de dinámicas } \\
\text { que faciliten las aportaciones } \\
\text { críticas y fundamentadas, así } \\
\text { como la formación de un } \\
\text { juicio propio, el cual permi- } \\
\text { ta la creación y recreación } \\
\text { de alternativas didácticas } \\
\text { innovadoras en el campo del } \\
\text { aprendizaje virtual. }\end{array}$ & $\begin{array}{l}\text { - Interacción en los foros en } \\
\text { línea } \\
\text { - Desarrollo de un entorno de } \\
\text { simulación para la construc- } \\
\text { ción de un curso en línea } \\
\text { - Construcción de estrategias } \\
\text { alternativas en evaluación } \\
\text { para los cursos virtuales }\end{array}$ \\
\hline $\begin{array}{l}\text { Wikis para el } \\
\text { trabajo } \\
\text { colaborativo } \\
(2009)\end{array}$ & $\begin{array}{l}\text { Propiciar el uso ade- } \\
\text { cuado de los sistemas } \\
\text { wiki por parte de los } \\
\text { profesionales que tie- } \\
\text { nen funciones docentes } \\
\text { dentro de la UNED } \\
\text { para propuestas de } \\
\text { trabajo colaborativo en } \\
\text { educación a distancia. }\end{array}$ & $\begin{array}{l}\text { La metodología es teórico- } \\
\text { práctica y la modalidad híbri- } \\
\text { da, pues para desarrollar } \\
\text { las actividades propuestas, } \\
\text { el participante pondrá en } \\
\text { práctica lo que aprenda en } \\
\text { las sesiones presenciales, así } \\
\text { como los conocimientos que se } \\
\text { van generando a partir de la } \\
\text { interacción con compañeros } \\
\text { y tutores. Se desarrollará un } \\
\text { trabajo colaborativo y un tra- } \\
\text { bajo individual. }\end{array}$ & $\begin{array}{l}\text { - Construcción de una wiki } \\
\text { - Realización de un trabajo } \\
\text { colaborativo en un sistema } \\
\text { wiki } \\
\text { - Interacción en los foros en } \\
\text { línea }\end{array}$ \\
\hline
\end{tabular}




\begin{tabular}{llll}
\hline Curso / Año & Objetivo o propósito & \multicolumn{1}{c}{$\begin{array}{c}\text { Estrategia } \\
\text { metodológica }\end{array}$} & Técnicas didácticas \\
\hline $\begin{array}{lll}\text { Pedagogía } \\
\text { universitaria }\end{array}$ & $\begin{array}{l}\text { Promover una práctica } \\
\text { de excelencia a partir }\end{array}$ & $\begin{array}{l}\text { Los participantes realizan activi- - Interacción en foros en } \\
\text { dades grupales e individuales con línea }\end{array}$ \\
para la & del Modelo Pedagógico & las cuales se abren espacios para - Reflexión sobre la normati- \\
educación a & y el Marco Estratégico & analizar y reflexionar a partir va institucional \\
distancia & en los equipos docentes & de nuestro Modelo Pedagógico, - Creación de ensayos \\
(2010) & el la UNED. & Marco de Estratégico, \\
& el Reglamento de Gestión \\
& Académica y otros documentos \\
& que nos permiten comprender \\
& cómo trabaja la UNED y cómo se \\
& visualiza el papel del estudiante. \\
& Del mismo modo, se cuenta con \\
& la participación de expertos de \\
& la Vicerrectoría en Investigación, \\
& Programa de Apoyo Curricular \\
& y Evaluación de los aprendiza- \\
& jes (PACE) y la Dirección de \\
& Producción Académica (DPA). \\
\hline
\end{tabular}

Fuente: Elaboración propia con base en los diseños didácticos de los cursos de la dependencia. Se reproducen fielmente lo señalado en los diseños.

Con base en lo observado en la tabla anterior, durante esta última etapa se interioriza en la práctica el Modelo Pedagógico de la Universidad como uno de los factores de éxito en los procesos de desarrollo profesional, pues junto con los facilitadores, los equipos de participantes se han propuesto construir conocimientos, a partir de su propia práctica en la educación a distancia.

En este sentido, se nota que hay una diferencia con los períodos anteriores, en relación con las estrategias utilizadas, pues actualmente, buscan una interacción más "horizontal" entre el tutor y los estudiantes y de los estudiantes entre ellos mismos, reflexionando sobre su práctica con situaciones desafiantes, por medio de las cuales resuelvan y apliquen lo aprendido.

Se puede afirmar que durante este período se consolida un proceso de desarrollo profesional iniciado desde 2005, el cual es concebido como lo señala Ruiz (1998):

Es un proceso de formación continua a lo largo de toda la vida profesional, que produce un cambio y un mejoramiento de las conductas docentes en las formas de pensar, valorar y actuar sobre la enseñanza. El desarrollo profesional implica procesos que originan cambios en la forma de pensar, de juzgar y, sobretodo, de actuar (p.35).

En el anterior sentido, los procesos de desarrollo profesional (atendiendo el concepto de Ruiz) que se desarrollan en el CECED durante este último período se puede indicar que son de naturaleza teórico-práctica y utilizan una metodología participativa, pues son los participantes quienes reflexionan sobre su propia práctica, las fortalezas, debilidades y necesidades tanto personales como profesionales en función del servicio que brindan promoviendo el aprender a aprender en los estudiantes. Esta naturaleza teóricopráctica requirió la comprensión de lo que es desarrollo profesional y sus diferencias con la capacitación "tradicional"; en este sentido, se cambiaron las estrategias de modo que no solo respondieran a ese aprendizaje para la vida, sino que fueran funcionales y significativas para los entornos virtuales. 
Señala Salas (2010) que por esto:

Se ha propuesto como estrategia preferente la aplicación de lo estudiado en casos concretos, mediante procesos de investigación, de reflexión, tanto sobre la teoría como de la práctica, y de análisis de situaciones concretas relacionadas con el diseño e implementación curricular para cursos en línea" (Salas, M. entrevista personal, 25 de abril de 2010).

Del mismo modo, los procesos de desarrollo profesional están dirigidos por un equipo de facilitadores del Centro de Capacitación de la UNED, quienes se encargan de dirigir todo el desarrollo: "diseñar y dar dirección a la mediación pedagógica, motivar a los participantes, presentar los foros de cada semana, atraer la atención de quienes no participan, valorar las participaciones, entre otras tareas" (Salas, M. entrevista personal, 25 de abril de 2010).

Los logros alcanzados en dichos procesos giran alrededor del intercambio de experiencias entre los diferentes participantes, lo cual es posible gracias a la discusión generada en las herramientas disponibles en los LMS. En la mayoría de las ocasiones, los participantes, a partir de las lecturas asignadas, cuentan sus vivencias: las limitaciones que se les presentaron, cómo las resolvieron, qué factores favorecieron su práctica y las situaciones que les impidieron mejorar. Otras veces, hay quienes hacen preguntas específicas sobre la temática tratada para resolver algún problema de los cursos que imparten y en la gran parte de los casos, estas personas reciben muchas posibilidades de solución.

En resumen, se puede afirmar, como señala Salas (2010) que:

Al conversar con docentes que han vivido estos procesos de desarrollo profesional, resulta muy satisfactorio escucharles decir que, a partir de ellos y alimentados por las relaciones académicas que siguen teniendo con el CECED, ellos han logrado:

Desarrollar un equipo de trabajo interdisciplinario, integrado y comprometido con el mejoramiento de los procesos de enseñanza-aprendizaje de la UNED.

- Establecer la innovación y la eficacia de los procesos de aprendizaje a distancia tradicional y en línea que desarrollan en sus cátedras.

- Diversificar las propuestas de evaluación de los aprendizajes y continuar con los procesos de capacitación (profundización).

- Rediseñar sus cursos en línea y elaborar objetos de aprendizaje (Salas, M. entrevista personal, 25 de abril de 2010)".

En suma, los principales logros de este desarrollo profesional en la actualidad se pueden resumir así:

Fortalecimiento de los procesos de formación con apoyo de los entornos virtuales, así como el énfasis en el uso pedagógico de estos.

Se repensó la cantidad de sesiones presenciales en los cursos y se disminuyeron cuando era muchas.

- $\quad$ Inicio de la oferta del curso Enseñar $\mathrm{y}$ aprender en entornos virtuales, como respuesta a la creciente necesidad de los equipos docentes de la UNED para atender los procesos en entornos virtuales y como respuesta a la solicitud del Consejo de Rectoría sobre ello.

- Creación y diversificación de los proyectos de capacitación anuales, ya no esporádicos como en períodos anteriores.

Por su lado, los participantes de cursos indicaron los siguientes aspectos de esta etapa: 
Tabla 6

Comentarios de los participantes en cursos del CECED durante el período 2007-2010.

\begin{tabular}{ll}
\hline \multicolumn{1}{c}{ Aspectos positivos } & Aspectos por mejorar \\
\hline - Calidad humana y & - Imposibilidad, a la \\
preparación académi- & fecha, de poder coor- \\
ca del grupo de facili- & dinar y adecuar, con \\
tadores. & mejores resultados, \\
- Contenidos actuales & la capacitación para \\
y aplicables, de fácil & tutores del SEP. \\
aprehensión. & - Excesiva diferen- \\
- Innovación en los & cia o heterogeneidad \\
usos de los recursos e & entre los participan- \\
inquietud por transmi- & tes de los cursos, en \\
tir y lograr su uso por & relación con su forma- \\
parte de los partici- & ción previa necesaria \\
pantes de los diferen- & para estos. Creo que \\
tes cursos. & impacta en la dinámi- \\
-Mejor desempeño pro- & ca del grupo y en los \\
fesional de mi perso- & aportes que se pue- \\
na, y contribuyendo & dan dar. \\
a la calidad de proce- & - La metodología \\
sos como tutora de la & empleada en las \\
UNED. Muy agrade- & sesiones presenciales. \\
cida. & - La obligatoriedad \\
- Un curso bien estruc- & de dar opiniones en \\
turado. & temas que a veces no \\
- Buen manejo de los & se desean abordar. \\
profesionales respon- & - No poder completar \\
sables. & el curso por razones \\
- Intercambio con com- & laborales y la impo- \\
pañeros/as de dife- & sibilidad de alter- \\
rentes lugares de la & nativas de parte del \\
UNED. & CECED. \\
\hline
\end{tabular}

Fuente: Ruiz, Warner (2010). Encuesta sobre la percepción de los participantes de los cursos ofrecidos por el CECED durante los años 2000-2010. Manuscrito no publicado.

\section{A modo de cierre}

Puede notarse en este breve recorrido que, durante los tres períodos desde su creación, el CECED se ha abocado a la promoción del desarrollo profesional de los equipos docentes de la Universidad, así como de instituciones nacionales (Universidad de Costa Rica, Instituto de Desarrollo Profesional Uladislao Gámez del Ministerio de Educación Pública de Costa Rica, Instituto Costarricense de Acueductos y Alcantarillados, Banco de Costa Rica, entre otros) e internacionales (Universidad Técnica Particular de Loja, Red Educ@L -Red de fortalecimiento de capacidades de educación virtual en América Latina-, auspiciada por InWent, Alemania; Universidad Nacional Autónoma de Nicaragua, entre otros), siempre con la firme intención de promover experiencias en los profesionales que impacten de manera positiva su práctica docente. Del mismo modo, lo ha hecho evolucionando desde propuestas pedagógicas de capacitación "tradicional" a una noción de desarrollo profesional mucho más vivencial, significativa, investigativa, crítica y colaborativa.

En este sentido, obsérvese la gráfica número uno donde se muestra la evolución de la oferta de capacitación del CECED, la cual se ha ajustado a las necesidades de los equipos docentes de la UNED.

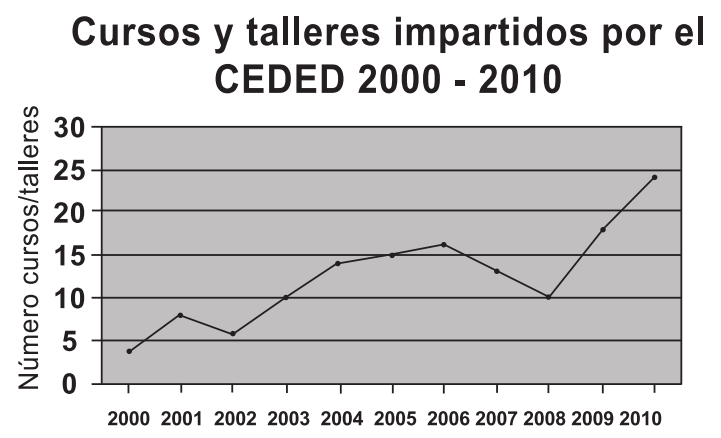

Fuente: Elaboración de Francisco Mora Vicarioli con base en datos aportados por el autor de los expedientes institucionales del CECED.

Por ejemplo, entre cursos, talleres y seminarios se han desarrollado:

La actual oferta de capacitación 2010 se compone de 24 procesos entre cursos y talleres que abarcan diferentes áreas de prioridad de la Universidad: el Modelo Pedagógico, la evaluación de los aprendizajes, la inclusión de las tecnologías de la información y la comunicación en los procesos educativos, investigación, entre otras. 
Es un reto para el CECED continuar sobre el camino iniciado, para ello es importante que se reflexione sobre lo siguiente:

- La implementación de lineamientos para la sistematización de las experiencias vividas en los procesos de desarrollo profesional que lleva acabo, pues si bien es cierto que se da un acompañamiento luego de finalizado el curso, este es a corto plazo.

Generar una normativa interna para la sistematización de la documentación (impresa y digital), pues una limitante en la presentación de este escrito es la ausencia continua de fuentes primarias durante los primeros años de creación del Centro y en algunos años del último período dado que los registros digitales o impresos no se ubicaron del todo.

La recopilación de programas de cursos, materiales impresos y digitales, insumos externos, percepciones de los participantes y evaluación académica del curso es un reto que los profesionales del CECED deben implementar con mayor rigurosidad pues permitirá aún más consolidar lo que en la práctica se hace: implementar procesos de desarrollo profesional que incidan significativamente en el participante y en la Institución.

Concretar ese proceso iniciado tal como lo cita Gradillas (2001): “(...) gestionar bien para crecer todos. Compartir para aprender. Aprender y, nuevamente compartir; compartir para volver de nuevo a aprender y alimentar, una y otra vez, la espiral interminable del conocimiento", pues el desarrollo profesional radica en esas experiencias compartidas y significativas que muchas veces se construyen de forma colectiva.

Por último, se puede señalar que el principal alcance luego de visualizar la evolución que se ha dado en los períodos indicados fue el inicio de la consolidación de un proceso de desarrollo profesional vertido o reflejado en los equipos docentes de la UNED que cursaron la oferta del Centro. La concepción de capacitación "tradicional" con la cual inició el CECED fue desplazada prácticamente desde la mitad del período en estudio, puesto que se implementaron propuestas que impactaron positivamente en las prácticas tutoriales, curriculares y evaluativas, imbuidas todas desde una perspectiva de desarrollo profesional cumpliendo con lo propuesto en la Misión y Visión de la UNED, es decir, consolidando la excelencia académica en los equipos docentes para que junto al actor fundamental del Modelo Pedagógico, el estudiante, se construya una sociedad más justa.

Asimismo, la importancia de este escrito radica, entre otras razones, en reflexionar sobre la escasez de investigaciones nacionales respecto al desarrollo profesional y las buenas prácticas que han iniciado algunas Instituciones; por ello es necesario que se propongan nuevos procesos investigativos que permitan conocer las condiciones teórico-prácticas bajo las cuales tienen éxito centros como el CECED en el desarrollo profesional de equipos docentes.

\section{Referencias bibliográficas}

Ander-Egg, E. (1993). La Planificación Educativa. Conceptos, métodos, estrategias y Técnicas para Educadores. Argentina: Editorial Magisterio del Río de la Plata.

Barnett, M., Keating, T., Harwood, W., y Saam, J. (2002). Using emerging technologies to help bridge the gap between university theory and classroom practice: Challenges and successes. School Science \& Mathematics, 102(6), 299-313. Recuperado de http://onlinelibrary.wiley.com/ doi/10.1111/j.1949-8594.2002. tb17887.x/pdf 
Borko, H. (2004). Professional Development and Teacher Learning: Mapping the Terrain.Educational Researcher. Recuperado de http://www.aera. net/uploadedFiles/Journals_and_ Publications/Journals/Educational_ Researcher/Volume_33_No_8/02_ ERv33n8_Borko.pdf

Castillo, T. (2010). Capacitación y Formación del Personal Académico. Plan de la Vicerrectoría Académica. San José: UNED.

CIDE. (2001). El aula reformada: buenas prácticas de actualización y buenas prácticas docentes en cinco paises latinoamericanos. Recuperado de http://www.reduc.cl/homereduc. nsf/?Open

Culp, K., Kanaya, T. y Light, D. (2005). Factors influencing outcomes from a technology focused professional development program. Journal of Research on Technology in Education, 37(3), 313-329.

Dede, C. (2006). Online professional development for teachers: Emerging models and methods. Cambridge, MA, EE.UU.: Harvard Education Press.

Dede, C., Ketelhut, D., Whitehouse, P., Breit, L. y McCloskey, E. (2009). A research agenda for online teacher professional development. Journal of Teacher Education 60, 1, 8-19.

Delors, J. (1996). La educación encierra un tesoro. Barcelona: Ediciones UNESCO.

Fallas, I., Arias, M., Villers, R., Escalante, M. y McCloskey, E. (2010). La puesta en práctica de una pedagogía innovadora en el desarrollo profesional en línea para educadores. En Memoria del XV Congreso Internacional de Tecnología y Educación a Distancia. San José, Costa Rica.

Gradillas, . (2001). Propuesta para la formulación de una estrategia de gestión del conocimiento. Recuperado de http:// www.gestiondelconocimiento.com/ documentos $2 /$ mgradillas/estrat.htm
Guskey, T. (2000). Evaluating professional development. Thousand Oaks, CA, EE.UU.: Corwin Press.

Huberman, S. (2005). ¿Cómo se forman los capacitadores? Arte y saberes de su profesión. Buenos Aires: Paidós.

Marzano, R. (2003). What works in schools: Translating research into action. Alexandria, VA: Association for Supervision and Curriculum Development.

Mora, D. (2000). Proyecto de Creación del CECED. Manuscrito no publicado. Vicerrectoría Académica, Universidad Estatal a Distancia.

Noguera, M., Fuentealba, R., Osandón, L., Portales, R. y Quiroga, P. (2002). Desarrollo Profesional Docente. Experiencias de Colaboración en la Enseñanza Media. Ministerio de Educación: Chile.

Robalino, M. (2004). Desarrollo profesional y humano de los docentes: Una responsabilidad social. En Flores, Isabel (2004). ¿Cómo estamos formando a los maestros en América Latina? (pp.159-168). Santiago de Chile, Chile: Oficina Regional de Educación de la UNESCO para América Latina y el Caribe.

Ruiz, J. (1998). ¿Cómo mejorar la institución educativa?. Santafé de Bogotá: Cooperativa Editorial Magisterio.

Ruiz,W. (2010). Encuesta sobre la percepción de los participantes de los cursos del CECED durante los años 2000 - 2010. Manuscrito no publicado.

Salas, M. (2009). De la capacitación al desarrollo profesional y a las comunidades virtuales de aprendizaje. Ponencia presentada en el X Congreso Internacional Virtual Educa, Argentina. Recuperado de http:// www.virtualeduca.info/ponencias/643/ Ponencia\%20Marianela\%20Salas.doc 